\title{
The Red MSX Source Survey - Massive Star Formation in the Milky Way
}

\author{
Stuart Lumsden ${ }^{1}$, Melvin Hoare ${ }^{1}$, Ben Davis ${ }^{1}$ and the RMS team ${ }^{2}$ \\ ${ }^{1}$ School of Physics and Astronomy, University of Leeds, Leeds, UK \\ email: s.l.lumsden@leeds.ac.uk \\ ${ }^{2}$ http://www.ast.leeds.ac.uk/RMS
}

\begin{abstract}
We present the results of a Galaxy-wide survey for young massive stars still in the process of formation. Our data are consistent with a model in which the stars form through accretion disks with the overall Galactic star formation rate being $3 M_{\odot}$ per year.
\end{abstract}

Keywords. Stars: formation, Stars: luminosity function, Galaxy: stellar content

We have used mid-infrared MSX and near-infrared 2MASS data to define a sample of massive young stellar objects (MYSOs) and compact HII regions (Lumsden et al. 2002). A programme of follow-up study has resulted in a final catalogue of about 500 of each type of object. We have successfully modelled the numbers we see in this population as a function of luminosity using an accretion based prescription for massive star formation (McKee \& Tan 2003), and find good agreement between the predicted and observed numbers of MYSOs and compact HII regions given the known MSX sensitivity. The model

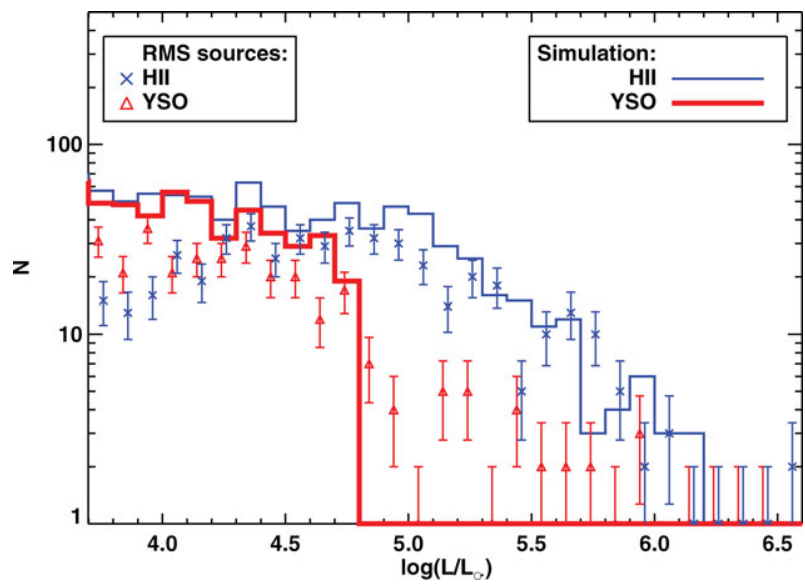

Figure 1. Luminosity distribution of the MYSOs (red points), compact HII regions (blue points) compared with the simulated population (solid lines)

\section{References}

Lumsden, S. L., Hoare, M. G., Oudmaijer, R. D., \& Richards, D. 2002, MNRAS 336, 621

McKee, C. F. \& Tan, J. C. 2003, ApJ 585, 850

Hosokawa, T. \& Omukai, K. 2009, ApJ, 691, 823 